\section{ambient \\ SCIENCE \\ Vol. 06h(1):56-57 \\ Year 2019}

NOTES ON CASE HISTORY

\title{
Botulism with LateSymptoms in Four Members of a Family
}

\section{Maryam Ziadi Lotfabadi, Seyed Reza Habibzadeh, Behzad Shahi, Parvin Zohoorian Sadr, Syyed Reza Ahmadi, Mahdi Foroughian*}

Department of Emergency Medicine, Mashhad University of Medical Sciences, Mashhad, Iran

\section{Introduction:}

Botulism is a life-threatening illness. The paralytic attacks due to the botulinum toxin quickly lead to death due to the cessation of breathing caused by respiratory muscle paralysis. Failure to detect the common contamination source of illnesses can spread rapidly and develop widespread epidemics with high mortality rates. Based on the guidelines for the prevention and control of diseases, the Ministry of Health and Medical Education in Iran has instructed to report telephonically whenever the botulism disease would be reported. According to the national guidelines, there is no need for laboratory conf irmation to start treatment by injection of polyvalent equine-derived botulinumantitoxin (A, B, E) (Raeisi et al., 2012)..

\section{Case history:}

A 47-year-old woman (mother of the family), a 27-year-old boy and two children aged 6 and 8 years (children of the family) residing in one of the villages in the North Khorasan Province, Iran, referred to the emergency department of Imam Reza (AS) Hospital with ptosis, dysphonia, dropped neck and asymmetric limb weakness. The patients with complain of limb weakness and flaccid paralysis was under different examinations (NCV, MCV, Brain CT scan etc.) in the city, and was referred to a higher center because of failure to detect and suspect of carbon monoxide poisoning.

The symptoms initiated progressively two weeks ago in the form of weakness, lethargy, ptosis, and dropped neck. The symptoms were more severe in children, they were unable to swallow and thus transferred to the ICU for care. The patients were under pulse oximetry during the entire period of hospitalization and were treated with a serial arterial blood gas (ABG) test for possible respiratory failure. None of the patients exhibited breathing problems and the need for respiratory support during admission. Other tested parameters viz., BUN, Cr, Na, K, LFT, and ECG were normal. The mother had paralytic ileus and was treated with lactulose and anti-hemorrhoid suppository for constipation. According to the National Protocol, an immediate report on the risk of botulism was given to the

\author{
Study Area: Mashhad, Iran \\ Coordinates: $36^{\circ} 18^{\prime} \mathrm{N} ; 59^{\circ} 36^{\prime} \mathrm{E}$
}

Key words: Foodborne botulism, Ptosis, Paraparesis, Clostridium botulinum

health center. Samples of stool, serum and suspected contaminated foods were sent to the National Reference Laboratory (Pasteur Institute of Iran) by observing the cold chain at a temperature of $4^{\circ} \mathrm{C}$, all of which were reported to be negative.

In all of our patients, the botulism was diagnosed based on clinical signs and history taking of consuming the same food, Duraq Yogurt (a type of yogurt stored in goatskin for extended periods in rural areas of Iran). The eldest son of the family lived in a place far away from other family members, who had traveled to the village just before the signs began, and had used Yogurt (a possible source of contamination).

Based on national guidelines, there is no need for laboratory approval to start treatment (Raeisi et al., 2012). Due to suspicious clinical symptoms and high diagnostic need, the patients were treated with the injection of polyvalent equine-derived botulinum antitoxin (A, B, E). The clinical symptoms were decreased with the onset of treatment. Eventually, the symptoms were disappeared and the patients were discharged from the hospital with a good general health condition.

\section{Discussion:}

Botulism occurs commonly by a Gram-positive bacterium Clostridium botulinum or, occasionally by C.butyricum, C.baratii or C.argentinese (Brola et al., 2013). Till date total eight types of botulinum toxin have been identified; toxins $\mathrm{A}, \mathrm{B}$, and $\mathrm{E}$ are the most common ones, whereas type $\mathrm{F}$ sometimes acts as an agent of disease in humans (Brola et al., 2013). The symptoms appear due to the release of acetylcholine in nerve terminals at neuromuscular junctions and synapses of the parasympathetic nervous system (Sobel, 2005).

The incubation period for botulism varies from a few hours to 16 days, whereas our patients visited and admitted after about 14 days. Neurological symptoms are the same in all types of botulism. In foodborne botulism, nausea, vomiting, abdominal pains are common in the first stages. The botulinum toxin causes constipation (Tabatabaee et al., 2006), although diarrhea can also be seen in some cases 
(Tabatabaee et al., 2006). Constipation and ileus were reported in one of our patients. The progression of the disease is associated with cranial nerve involvement, descending flaccid paralysis in motor and autonomic nerves, which were also reported in our patients. The symptoms are usually bilateral, but sometimes asymmetric, especially in Wound Botulism (http://www.cfsph.iastate.edu/DiseaseInfo/ disease.php? name=botulism\&lang=en). Common symptoms include blurred vision, diplopia, photophobia, ptosis, expressionless, slow and slash speech, dysphagia, dysuria, dry eye syndrome, xerostomia, drowsiness and muscle weakness (Passaro et al., 1998; Brola et al., 2013).

The respiratory failure caused by botulism occasionally causes the patient to attach to ventilator for weeks, and the need for invasive and intensive care. The paralysis gradually resolves after several weeks, and early detection can lead to treatment with equine-derived antitoxin. Antitoxin inhibits the function of the toxin in the bloodstream and prevents the progression of the disease (Passaro et al., 1998). Emetic drugs, gastric lavage or enema can be used to eliminate contaminated food in the intestines (Raeisi et al., 2012; Underwood et al., 2007). Progression of the disease was discontinued in our patients with the onset of treatment, and the symptoms were gradually eliminated.

The most common complications of botulism are aspiration pneumonia and urinary tract infection, and death occurs in $7 \%$ to $10 \%$ of cases (Sobel, 2005). Early diagnosis should be based on earlier symptoms and clinical examination. The likelihood of diagnosis increases by an earlier record of taking home-cooked or canned common foods and examining similar symptoms in such individuals (https://www.who.int/csr/delibepidemics/botulism/en/). The presence of similar symptoms in a family led us to diagnose the disease that was identified after further evaluation of their common food. In an earlier report of Hosseini et al. (2010) on 131 samples of Iranian traditional food ( 57 cheese samples, 11 Kashk samples and 63 smoked fish samples) using standard monovalent antitoxin and bioassay, they found the botulinum toxin in $4.5 \%$ of the samples. In this study, C.botulinum type A and $\mathrm{E}$ were more common in food samples. Local yogurt was a common source of food in our patients.

According to the Ministry of Health, the actual prevalence and distribution of illness in Iran is still unknown. The first epidemic of this disease occurred in northern Iran in 1965 and sporadic cases or regional epidemics occur in different parts of the country during recent years. The most important cause of illness in Iran is the consumption of seafood, and then bag cheese, homemade Kashk, canned Tuna fish and canned Olive imported (Raeisi et al., 2012). According to the National Protocol, the key intervention for a patient with botulism is the positioning of disease in the differential diagnosis of poisoning; in case of suspicion, the patient must be referred immediately to an equipped hospital for admission. Samples of used food suspected of having a toxin along with stool or serum samples should be sent to the referred laboratory. All people who have had a common food source with the patient should be examined and screened for signs and symptoms. Contaminated food should be discarded after boiling, and the cans must be buried at high depths (Raeisi et al., 2012).

Acknowledgements:

Thanks are due for all such medical care staff who took part in this study.

\section{References:}

Brola, W., Fudala, M., Gacek, S. \& Gruenpeter, P. (2013): Foodborne botulism: still actual topic. BMJ Case Rep., bcr201200779.

Hosseini, H., Tavakoli, H.R., Meshgi, M.A., Khaksar, R., Hosseini, M. \& Khakpour, M. (2010): Survey of Clostridium botulinum toxins in Iranian traditional food products. Comp. Clin. Pathol., 19(3): 247-250.

Passaro, D.J., Werner, S.B., McGee, J., Mac Kenzie, W.R. \& Vugia, D.J. (1998): Wound botulism associated with black tarheroin among injecting drug users J. Am. Med. Asso., 279(11):859863.

Raeisi, A., Zahrayei, S.M., Najafabadi, M.S. (2012): National Communicable Diseases Operation Plan in Disaster and Emergencies. Pub. by: Ministry of Health and Medical Education. Center for Disease Management, 1391.

Tabatabai, M., Zahraei, M.H.A., Ghotbi, M. \& Rahimi, F. (2006): Principles of prevention and treatment of communicable diseases. Mir-Younes SM. 2006:1-9

Sobel, J. (2005): Botulism. Clin. Infect. Dis., 41(8):1167-1173.

Underwood, K., Rubin, S., Deakers, T. \& Newth, C. (2007): Infant Botulism: A 30-Year Experience Spanning the Introduction of Botulism Immune Globulin Intravenous in the Intensive Care Unit at Children's Hospital Los Angeles. Pediatrics, 120(6):1380-1385. 